\title{
Capacity Performance Analysis of Coherent Detection in Correlated Fading Channels Using Finite State Markov Models
}

\author{
Parastoo Sadeghi and Predrag Rapajic \\ School of Electrical Engineering and Telecommunications \\ The University of New South Wales \\ Sydney NSW 2052 Australia
}

\begin{abstract}
In this paper, an information theoretic approach is taken to the problem of joint channel estimation and coherent data detection in time varying flat fading channels. At the receiver side, the fading phase process is partitioned or mapped into a finite state Markov channel (FSMC). Using simulation-based capacity analysis of FSMC models, accurate capacity calculation of time varying fading channels is carried out and the effect of number of channel phase partitions on the channel capacity performance is studied. In BPSK scheme, the capacity is saturated beyond 8 to 16 channel phase partitions. In QPSK signalling, the channel capacity gap between 16/32 channel phase partitions and 128 channel phase partitions is less than $0.3 \mathrm{~dB}$. Moreover, the capacity performances of BPSK and QPSK in unknown correlated fading are compared. The comparison reveals that to maintain the superior QPSK capacity performance, care must be taken in the choice of average transmitted power and symbol duration.
\end{abstract}

\section{INTRODUCTION}

Estimation of the fading channel phase is essential for the coherent detection of constant-envelope phase modulated signals. Phase-locked loop (PLL) devices are often used for the estimation of unknown channel phase in very slowly fading channels [1]. However, PLL devices fail to perform as desired in low SNR and relatively fast time varying fading conditions. Consequently, alternative channel estimation techniques should be implemented at the receiver side, for reliable coherent detection.

In this paper, we take the channel information capacity as the measure to obtain an equivalent finite state Markov channel (FSMC) model for flat fading channel phase estimation at the receiver side. FSMC models have been extensively used in the past for three different applications: 1) low-complexity simulation of the flat fading amplitude process [2]-[5], 2) simulation of packet errors in wireless data protocols [6], [7], and 3) estimation of the flat fading amplitude at the receiver side [8]-[10]. With a few exceptions [11], [12], applying the FSMC model for channel phase estimation and capacity performance analysis of the time varying fading channel is less recognized. It has to be emphasized that in our approach, the continuous-level nature of the channel is observed and taken into account at the receiver side, when the main parameters of the FSMC model are derived from the fading channel.

When the FSMC model is applied for channel estimation, the channel parameter to be estimated is mapped into a number of partitions or states. Since the FSMC model complexity increases with the number of channel states, it is worthwhile to optimize the number of channel phase partitions for accurately representing and estimating the fading phase process without sacrificing the receiver performance. High channel phase estimation accuracy is often assumed to be an essential requirement for the coherent detection [1, pp. 338-339]. The results of channel capacity analysis in Section IV contradict the expectations. In fact, the required resolution for the channel phase estimation is far less than what is implemented in practice, both in very slow and relatively fast fading channels. For instance, the capacity of BPSK and QPSK schemes is saturated beyond $8 / 16$ and $16 / 32$ channel phase partitions, respectively.

Furthermore, we have compared the capacity performance of BPSK and QPSK schemes in time varying fading channels. The analysis provides insight into the actual performance of QPSK and BPSK under different average transmitted power and symbol duration conditions. The results indicate that in relatively fast time varying fading channels, QPSK information rate can be almost double the BPSK information rate, only at the expense of doubling the average transmitted power.

The paper is organized as follows. In Section II of the paper, the system model is reviewed and the notations are set out. Section III presents the FSMC modelling of the flat fading phase process. Numerical results are presented in Section IV. Section V concludes the paper.

\section{System MODEL}

Throughout the paper, all signals are represented in their complex, low-pass format, with in-phase and quadrature components denoted by $I$ and $Q$ subscripts, respectively. A continuous-time signal $w(t)$ is sampled at the receiver side with symbol interval $T$, and is presented as $w_{k}=w(k T)$. Discrete-time sequences will be denoted as vectors $w_{k}^{l}=$ $\left[w_{k}, w_{k+1}, \ldots, w_{l}\right]$. Random variables or processes and their realizations are referred to by upper case and corresponding lower case letters, respectively. The probability density function (pdf) of the random variable $W$ is shown by $f(W=w)$, and is shortened to $f(w)$, where the context is clear.

Following Clarke's model for flat fading channels [13], unknown, time varying amplitude and phase distortions are 
introduced to the transmitted signal. If the channel fading changes slowly enough to be assumed constant over the $k^{\text {th }}$ signalling interval, the complex, discrete-time, M-ary PSK received signal is written as

$$
r_{k}=r_{I k}+j r_{Q k}=\sqrt{\mathcal{E}_{s}} c_{k} \mathrm{e}^{j \phi_{k}}+n_{k} .
$$

In (1), $n_{k}$ is a sample function from the complex, additive white Gaussian noise (AWGN) process, with variance per dimension equal to $\frac{N_{0}}{2}, \mathcal{E}_{s}$ is the symbol energy and the equiprobable information symbols $b_{k}=m, m \in$ $\{0,1, \cdots, M-1\}$, modulate the phase as $\phi_{k}=\frac{2 \pi m}{M}$.

Channel fading gain $c_{k}=c_{I k}+j c_{Q k}=a_{k} e^{j \theta_{k}}$, is a zero-mean stationary complex Gaussian process, which can be represented in polar format by fading amplitude $a_{k}$, and fading phase $\theta_{k}$. The distribution of the fading amplitude $a_{k}$ is Rayleigh, given by

$$
f\left(A_{k}=a_{k}\right)=\frac{a_{k}}{\sigma^{2}} \exp \left(-\frac{a_{k}^{2}}{2 \sigma^{2}}\right), \quad 0 \leq a_{k}<\infty,
$$

where $\sigma^{2}$ is the fading variance per dimension (normalized to be 0.5 ). The fading phase is uniformly distributed between 0 and $2 \pi$. The channel dynamics is completely characterized by the auto-correlation function (ACF) of the fading process

$$
\rho_{m}=\sigma^{2} J_{0}\left(2 \pi m f_{D} T\right), \quad 0 \leqslant m<\infty,
$$

where $J_{0}$ is the zero-order Bessel function of the first kind, and $f_{D}$ is the maximum Doppler frequency.

The distribution of the received signal conditioned on the fading amplitude $a_{k}$, fading phase $\theta_{k}$, and information modulated phase $\phi_{k}$ is written as

$$
\begin{aligned}
& f\left(r_{k} \mid a_{k}, \theta_{k}, \phi_{k}\right)=\frac{1}{\pi N_{0}} \\
& \exp \left(-\frac{\left(r_{I k}-\sqrt{\mathcal{E}_{s}} a_{k} \cos \left(\theta_{k}+\phi_{k}\right)\right)^{2}}{N_{0}}\right) \\
& \exp \left(-\frac{\left(r_{Q k}-\sqrt{\mathcal{E}_{s}} a_{k} \sin \left(\theta_{k}+\phi_{k}\right)\right)^{2}}{N_{0}}\right) .
\end{aligned}
$$

In order to determine the effect channel amplitude estimation on the receiver performance in unknown time varying fading channels, two ideal cases are examined and compared. In the first case, the channel capacity upper bound is computed when the receiver has perfect channel phase and amplitude CSI. The capacity with perfect phase and amplitude CSI is written as

$$
\begin{aligned}
& C_{\mathrm{CSI}}=h\left(r_{k} \mid a_{k}, \theta_{k}\right)-h\left(r_{k} \mid a_{k}, \theta_{k}, \phi_{k}\right) \\
& E\left\{\log f\left(r_{k} \mid a_{k}, \theta_{k}\right)\right\}-E\left\{\log f\left(r_{k} \mid a_{k}, \theta_{k}, \phi_{k}\right)\right\},
\end{aligned}
$$

where $h$ is the entropy function and $f\left(r_{k} \mid a_{k}, \theta_{k}, \phi_{k}\right)$ was given in (4). $f\left(r_{k} \mid a_{k}, \theta_{k}\right)$ is determined by proper summation of $f\left(r_{k} \mid a_{k}, \theta_{k}, \phi_{k}\right)$ over all possible $\phi_{k}$. In the second case, the receiver is only provided with perfect phase CSI and has no access to amplitude CSI. The channel capacity is written as

$$
\begin{aligned}
& C_{\mathrm{Ph}-\mathrm{CSI}}=h\left(r_{k} \mid \theta_{k}\right)-h\left(r_{k} \mid \theta_{k}, \phi_{k}\right) \\
& E\left\{\log f\left(r_{k} \mid \theta_{k}\right)\right\}-E\left\{\log f\left(r_{k} \mid \theta_{k}, \phi_{k}\right)\right\} .
\end{aligned}
$$

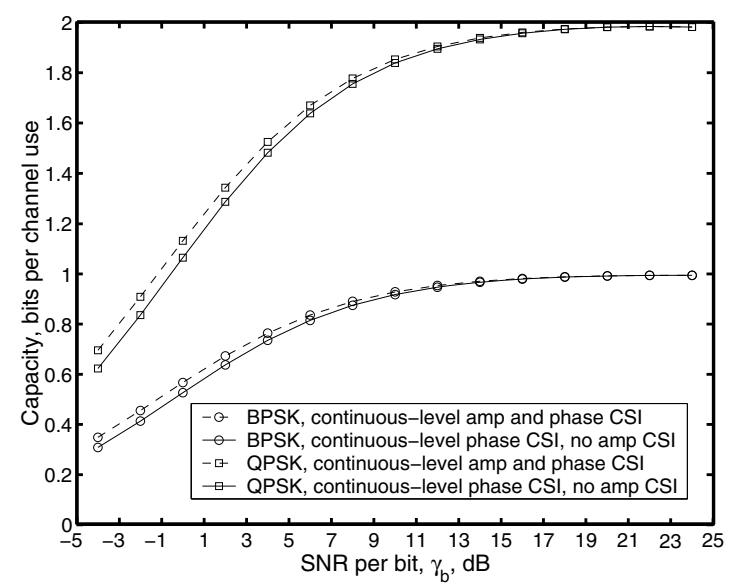

Fig. 1. Comparison of the channel capacity with and without channel amplitude CSI in BPSK and QPSK schemes. There is little gain in the perfect knowledge of the fading amplitude.

The results of calculating (5) and (6) are shown in Fig. 1 for BPSK and QPSK schemes. The striking point about this figure is that there is less than $0.8 \mathrm{~dB}$ gain in having access to perfect channel amplitude CSI, as compared to no channel amplitude CSI. The gap only exists in the low SNR region. In high SNR conditions, the knowledge of channel amplitude has almost no effect on the achievable information rate. On the other hand, it can be easily verified that the channel capacity with no phase information at the receiver side is zero. Recognizing the greater challenge of channel phase estimation for coherent detection of M-ary PSK schemes, the next section reviews the FSMC modelling of the fading phase process and the FSMC channel capacity.

\section{FSMC Modelling of the Flat FAding Phase}

The concept of FSMC modelling of the phase process at the receiver side is briefly explained here. The actual physical fading channel is following the Clarke's model, which was described in Section II. No approximation of the channel itself is carried out. In other words, the channel gain is continuouslevel and correlated with its infinite past, as given in (3). However, the receiver is is trying to estimate the channel phase in a partitioned version and also track the dynamics of the channel with finite channel memory order. Fig. 2 shows channel phase partitioning into 8 states. In a partitioned channel phase estimation scheme, the receiver tries to estimate the channel phase partition or state rather than continuous-level channel phase. Clearly, when the number of channel phase partitions is too high, phase partitions become narrow and the receiver is effectively estimating almost continuous-level channel phase. In general, the channel phase is partitioned into $L$ disjoint equiprobable regions. The FSMC model is said to be in state or partition $S_{k}=l$, when the channel phase is in the $\frac{\pi(2 l-1)}{L} \leqslant \theta_{k}<\frac{\pi(2 l+1)}{L}$ region.

Channel phase state transition probability in the FSMC 
model is denoted by $\mathrm{P}_{i j}$, and is given as

$$
\begin{aligned}
P_{i j} & =\operatorname{Pr}\left(S_{k+1}=j \mid S_{k}=i\right) \\
& =\frac{L}{2 \pi} \int_{\frac{\pi(2 i-1)}{L}}^{\frac{\pi(2 i+1)}{L}} \int_{\frac{\pi(2 j-1)}{L}}^{\frac{\pi(2 j+1)}{L}} f\left(\theta_{k+1}, \theta_{k}\right) d \theta_{k+1} d \theta_{k}
\end{aligned}
$$

and defining $\delta \triangleq \rho_{1} \cos \left(\theta_{k+1}-\theta_{k}\right), f\left(\theta_{k+1}, \theta_{k}\right)$ is [14]

$$
f\left(\theta_{k+1}, \theta_{k}\right)=\frac{\left(1-\rho_{1}^{2}\right)}{4 \pi^{2}} \frac{\sqrt{\left(1-\delta^{2}\right)}+\delta\left(\pi-\cos ^{-1}(\delta)\right)}{\sqrt{\left(1-\delta^{2}\right)^{3}}} .
$$

The probability of the received signal, conditioned on channel phase state $s_{k}$, and symbol phase $\phi_{k}$ is denoted by

$$
\begin{aligned}
& f\left(r_{k} \mid S_{k}=i, \phi_{k}\right)= \\
& \frac{L}{2 \pi} \int_{\frac{\pi(2 i-1)}{L}}^{\frac{\pi(2 i+1)}{L}} \int_{0}^{\infty} f\left(r_{k} \mid a_{k}, \theta_{k}, \phi_{k}\right) f\left(a_{k}\right) d a_{k} d \theta_{k},
\end{aligned}
$$

where $f\left(r_{k} \mid a_{k}, \theta_{k}, \phi_{k}\right)$ was defined in (4).

Due to the constellation symmetry in M-ary PSK scheme, the capacity of FSMC model is achieved when the the information symbols are equiprobable $\left(\operatorname{Pr}\left(\phi_{k}=\frac{2 \pi m}{M}\right)=\frac{1}{M}, \quad 0 \leqslant\right.$ $m \leqslant M-1)$, and the capacity is written under this input constraint as

$$
C_{\mathrm{FSMC}}=\lim _{n \rightarrow \infty} \frac{1}{n}\left\{h\left(r_{1}^{n}\right)-h\left(r_{1}^{n} \mid \phi_{1}^{n}\right)\right\} .
$$

In order to isolate the effect of channel phase partitioning from signal partitioning, the received signal $r_{k}$ is treated in its continuous-level format. There is no closed-form formula for the distribution of $f\left(r_{1}^{n} \mid \phi_{1}^{n}\right)$, and direct calculation of (10) is also computationally impossible for continuous-level signals. An efficient method is the numerical capacity analysis of (10) [15], [16]. Shannon-McMillan-Breimann theorem [17] for stationary ergodic processes states that

$$
\lim _{n \rightarrow \infty} \frac{-1}{n} \log f\left(r_{1}^{n} \mid \phi_{1}^{n}\right) \longrightarrow \lim _{n \rightarrow \infty} \frac{h\left(r_{1}^{n} \mid \phi_{1}^{n}\right)}{n},
$$

with probability 1. $f\left(r_{1}^{n} \mid \phi_{1}^{n}\right)$ is efficiently calculated using the BCJR algorithm [18] by defining $\alpha_{n}(i) \triangleq f\left(r_{1}^{n}, S_{n}=i \mid \phi_{1}^{n}\right)$, and the recursive formula

$$
\alpha_{k+1}(j)=\left[\sum_{i=0}^{L-1} \alpha_{k}(i) P_{i j}\right] f\left(r_{k+1} \mid S_{k+1}=j, \phi_{k+1}\right),
$$

where $P_{i j}$ was defined in (7) and $f\left(r_{k+1} \mid S_{k+1}=j, \phi_{k+1}\right)$ was given in (9).

The FSMC capacity upper bound is obtained when the receiver has a priori information about the channel phase state $s_{k}$ at each instant of time. In this case, the FSMC capacity is simplified to

$$
C_{\mathrm{FSMC}-\mathrm{CSI}}=h\left(r_{k} \mid s_{k}\right)-h\left(r_{k} \mid s_{k}, \phi_{k}\right) .
$$

\section{NumericAl RESUlts}

Numerical results for the capacity performance of BPSK and QPSK coherent detection in time varying fading channels are provided. As mentioned in Section III, the receiver estimates channel phase partitions and tracks the dynamics of the channel with a first-order memory Markovian assumption. Therefore, a FSMC equivalent of the fading channel is formed at the receiver side, using the procedure explained in the previous section.

Fig. 3 shows the FSMC capacity when the receiver has perfect knowledge about the channel phase partition where the channel phase falls on. However, the actual value of the channel phase within the phase partition is not disclosed to the receiver. Phase CSI assumption is accurate for very slow fading channels. The capacity was calculated using (13) for $L=2$ to $L=16$ channel phase partitions. The ultimate upper bound is the channel capacity with perfect continuouslevel phase information, given in (6). It is observed from this figure that 2 phase partitions in BPSK and 4 phase partitions in QPSK schemes are very coarse and do not result in acceptable capacity performance. However, 4 phase partitions in BPSK and 8 phase partitions in QPSK show acceptable capacity performance. Surprisingly, the perfect phase CSI bound is very closely achieved with only 8 and 16 channel phase partitions in BPSK and QPSK, respectively. This is equivalent to $\frac{2 \pi}{8}=\frac{\pi}{4}$ phase estimation resolution for BPSK and $\frac{2 \pi}{16}=\frac{\pi}{8}$ phase estimation resolution for QPSK. Based on these results, it is clearly concluded that a very simple FSMC model, with small number of channel states can replace PLL devices in very slow fading conditions. An immediate advantage of using FSMC Markov model for channel estimation is that soft outputs are readily assigned to channel phase states and joint iterative channel estimation and data detection [11], [12] can be implemented at the receiver side, which gives promising results in low SNR conditions. It is also observed from this figure that in very slow fading conditions, QPSK capacity is twice the BPSK capacity for a fixed SNR per bit. The capacity performance of BPSK and QPSK schemes will be compared in relatively fast, time varying channels in subsequent figures.

Fig. 4 shows the capacity performance of BPSK scheme in a time varying fading channel. The normalized fading rate is $f_{D} T=0.0125$. The number of channel phase partitions varies from 2 to 32. It is observed form the figure that there is not much gain in estimating the channel with 32 phase partitions, compared to 16 phase partitions. For example, at $\mathrm{C}=0.5$ bits/channel use, there is only $0.2 \mathrm{~dB}$ gain in channel phase estimation with 32 phase partitions. Moreover, it is observed that the first-order Markovian assumption is very accurate and the gap between the CSI bound and the FSMC capacity with 16 states at $f_{D} T=0.0125$ is less than $1 \mathrm{~dB}$ across the SNR region.

Fig. 5 shows the capacity in BPSK scheme at the normalized fading rate of $f_{D} T=0.025$. The number of channel phase partitions varies from 2 to 128. Similar to the results in Fig. 4 , there is not much gain in estimating the channel with 128 phase levels, compared to 16 phase partitions. For example, 
at $\mathrm{C}=0.5$ bits/channel use, there is only $0.3 \mathrm{~dB}$ gain in 128 level channel phase estimation, compared to channel phase estimation with 16 partitions. Moreover, it is observed that the first-order Markovian assumption is still relatively accurate and the gap between the FSMC CSI bound and the FSMC capacity with 16 states at $f_{D} T=0.025$ is less than $2 \mathrm{~dB}$ across the SNR region.

The capacity performance of QPSK in time varying fading channels is shown in Fig. 6. The normalized fading rate is $f_{D} T=0.025$. From this figure, it is observed that 32 channel phase partitions is accurate and there is no further need for finer channel phase estimation. Compared to the BPSK scheme at the same fading rate in Fig. 5, the gap between FSMC capacity and CSI capacity bound is wider and can be as high as $3.5 \mathrm{~dB}$ in high SNR region.

Next, we compare the capacity performance of BPSK and QPSK in time varying fading channels in a unified framework. In static or very slow fading channels, BPSK and QPSK are usually compared at the same energy per bit $\mathcal{E}_{b}=P T_{b}$, where $P$ is average transmitted power and $T_{b}$ is bit duration $\left(T=T_{b} \log _{2} M\right)$. However, in time varying fading channels, the choice of symbol period is also important, as it affects the normalized fading rate $f_{D} T$.

We study three different scenarios here. In the first case, BPSK and QPSK schemes are compared at the same energy per bit $\mathcal{E}_{b}=P_{B P S K} T_{b 1}=P_{Q P S K} T_{b 2}$, and at the same symbol period $T$ to keep the normalized fading rate $f_{D} T$ similar for both modulations. Equal symbol period dictates that the $T_{b 2}=\frac{T_{b 1}}{2}$. Hence, the energy per bit cannot be the same, unless $P_{Q P S K}=2 P_{B P S K}$. In the second case, the modulations are compared with the same transmitted power and at the same energy per bit $\left(P_{Q P S K}=P_{B P S K}\right.$, and $T_{b 2}=T_{b 1}$ ). However, this results in the QPSK symbol period being twice the BPSK symbol period. As a result, QPSK will experience double the fading rate as BPSK. In the third scenario, $P_{Q P S K}=P_{B P S K}$, while $T_{b 2}=\frac{T_{b 1}}{2}$. BPSK and QPSK undergo the same fading conditions. However, QPSK energy per bit is half of BPSK energy per bit.

The comparison results for three different cases mentioned above is shown in Fig. 7. As expected, if the QPSK average transmitted power is allowed to be double the BPSK average transmitted power, then the superior QPSK capacity performance over BPSK is maintained and the QPSK capacity is almost double the BPSK capacity. However, if the average transmitted power is limited to be the same for both modulations, QPSK capacity will no longer be twice the BPSK capacity and care must be taken in choosing the bit energy and symbol duration, in order to maintain the QPSK superior performance over BPSK. From Fig. 7, it is observed that at low SNR region, the emphasis should be on maximizing QPSK energy per bit, while letting QPSK experience faster fading rates. Whereas, in high SNR regime, it is vital to experience minimum fading rate by letting the QPSK energy per bit drop to half of BPSK energy per bit.

\section{CONCLUSIONS}

We proposed FSMC modelling for the capacity analysis of coherent detection in correlated time varying fading channels. The FSMC information capacity was used as the criterion to obtain the optimum number of channel phase partitions, while maintaining practical FSMC model computational complexity. The optimum phase partitioning resolution is 4 to 8 times the number of symbols in BPSK and QPSK schemes. Moreover, BPSK and QPSK capacity performance in time varying fading channels was compared under different transmitted power and symbol period assumptions. For similar average transmitted power in BPSK and QPSK, QPSK energy per bit should be maximized in low SNR regions and symbol duration should be minimized in high SNR conditions.

\section{REFERENCES}

[1] J. G. Proakis, Digital Communications, forth ed. New York: Mc Graw Hill, 2000.

[2] H. Wang and N. Moayeri, "Finite state Markov channel-A useful model for radio communication channels," IEEE Trans. Veh. Technol., vol. 44, no. 1, pp. 163-171, Feb. 1995.

[3] P. Bergamo, D. Maniezzo, A. Giovanardi, G. Mazzini, and M. Zorzi, "Improved Markov model for Rayleigh fading envelope," Electronic Letters, vol. 38, no. 10, pp. 477-478, May 2002

[4] J. Arauz, "Discrete Rayleigh fading channel modeling," 2002. [Online] Available: http://www.sis.pitt.edu/ jarauz/research/researchind.html

[5] Y. L. Guan and L. F. Turner, "Generalised FSMC model for radio channels with correlated fading," IEE Proc. Commun., vol. 146, no. 2 , pp. 133-137, Apr. 1999.

[6] M. Zorzi, R. R. Rao, and L. B. Milstein, "ARQ error control for fading mobile radio channels," IEEE Trans. Veh. Technol., vol. 46, no. 5, pp. 445-455, May 1997.

[7] Q. Zhang and S. Kassam, "Finite state Markov model for Rayleigh fading channels," IEEE Trans. Commun., vol. 47, no. 11, pp. 16881692, Nov. 1999.

[8] H. Kong and E. Shwedyk, "Sequence detection and channel estimation over finite state Markov channels," IEEE Trans. Veh. Technol., vol. 48, no. 3, pp. 833-839, May 1999.

[9] M. Riediger and E. Shwedyk, "Communication receivers based on Markov models of the fading channel," IEE Proc. Commun., vol. 150, no. 4 , pp. 275-279, Aug. 2003.

[10] L. Li and A. J. Goldsmith, "Low-complexity mamximum-likelihood detection of coded signals sent over finite state Markov channels," IEEE Trans. Commun., vol. 50, no. 4, pp. 524-531, Apr. 2002.

[11] P. Sadeghi, V. Trajkovic, and P. B. Rapajic, "Implicit and explicit receiver training in flat fading channels modeled as finite state Markov processes," in Proc. Int. Symp. on Inform. Theory (ISIT), Yokohama, Japan, 2003.

[12] C. Komninakis and R. D. Wesel, "Joint iterative channel estimation and decoding in flat correlated Rayleigh fading," IEEE J. Select. Areas Commun., vol. 19, no. 9, pp. 1706-1717, Sept. 2001.

[13] R. H. Clarke, "A statistical theory of mobile-radio reception," Bell Syst. Tech. J., vol. 47, no. 6, pp. 957-1000, 1968.

[14] W. C. Y. Lee, Mobile Communications Engineerings, 1st ed. New York: Mc Graw Hill, 1982.

[15] D. Arnold and H. Loeliger, "On the information rate binary-input Markov channels with memory," in IEEE Int. Conf. Commun.(ICC), Helsinki, Finland, 2001, pp. 2692-2695.

[16] A. Kavcic, "On the capacity of Markov sources over noisy channels," in IEEE Global commun. Conf. (GLOBECOM), San Antonio, TX, 2001, pp. 2997-3001.

[17] T. M. Cover and J. A. Thomas, Elements of Information Theory, 1st ed. New York: Wiley, 1991

[18] L. R. Bahl, J. Coke, F. Jelinek, and J. Raviv, "Optimal decoding of linear codes for minimizing symbol error," IEEE Trans. Inform. Theory, vol. 20, no. 3, pp. 284-287, Mar. 1974. 


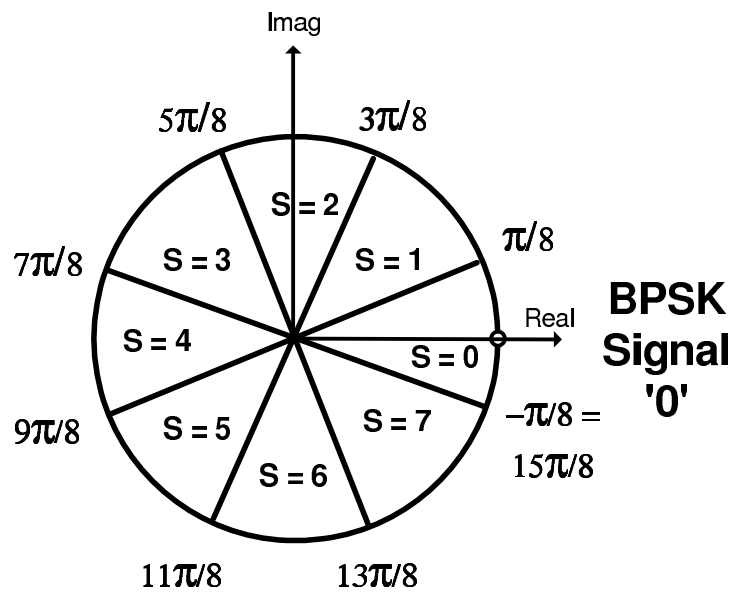

Fig. 2. Partitioning of the channel phase into 8 equiprobable disjoint regions, at the receiver side.

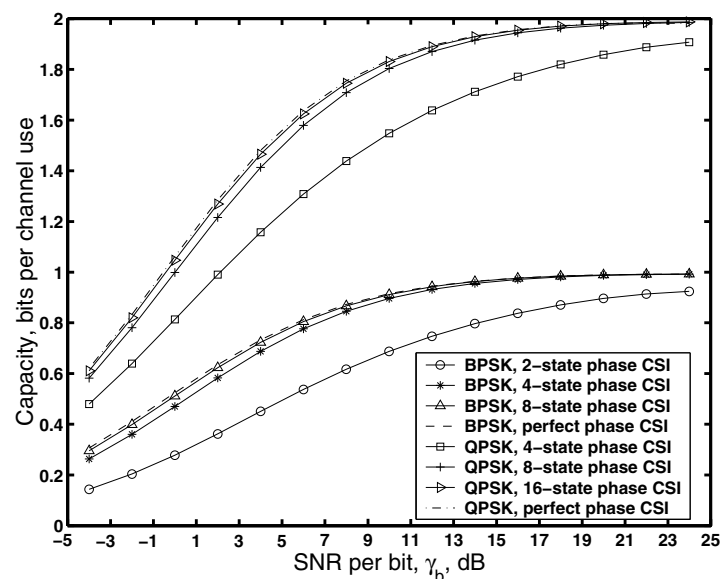

Fig. 3. FSMC capacity with partitioned phase CSI at the receiver side. The continuous-level perfect phase CSI is closely achieved with only 8 and 16 channel phase partitions in BPSK and QPSK, respectively.

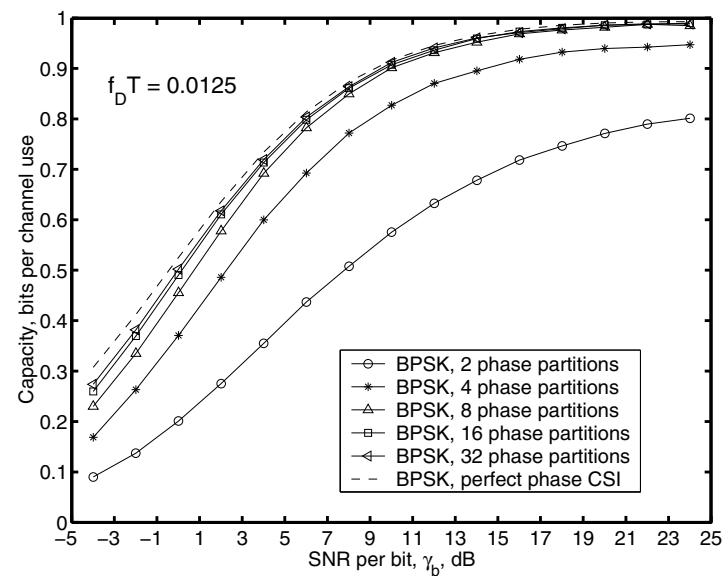

Fig. 4. FSMC capacity with 2 to 32 channel phase partitions in BPSK scheme, at $f_{D} T=0.0125$. The capacity is rapidly saturated with more than 16 channel phase partitions. The first-order Markovian assumption is accurately applicable for $f_{D} T=0.0125$.

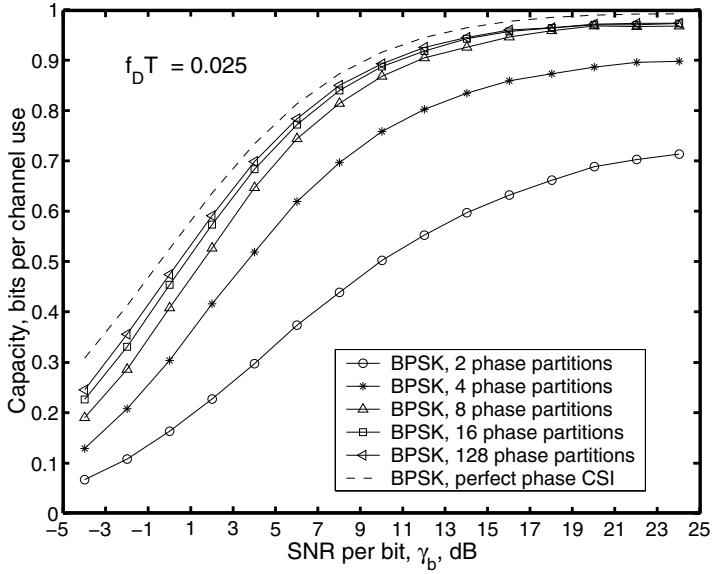

Fig. 5. FSMC capacity with 2 to 128 channel phase partitions in BPSK scheme, at $f_{D} T=0.025$. 128-level channel phase estimation is only $0.3 \mathrm{~dB}$ superior to channel phase estimation with 16 partitions at $\mathrm{C}=0.5 \mathrm{bits} / \mathrm{channel}$ use.

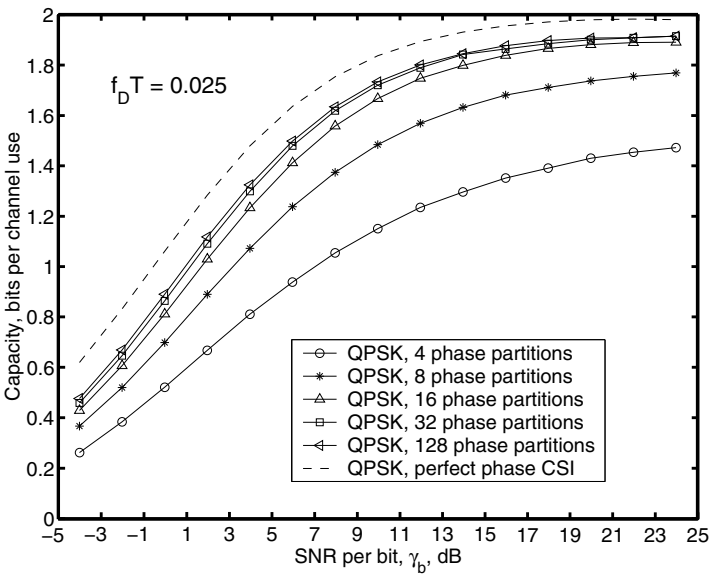

Fig. 6. FSMC capacity with 4 to 128 channel phase partitions in QPSK scheme, at $f_{D} T=0.025$. 128-level channel phase estimation is only 0.25 $\mathrm{dB}$ superior to channel phase estimation with 32 phase partitions at $\mathrm{C}=1$ bit/channel use.

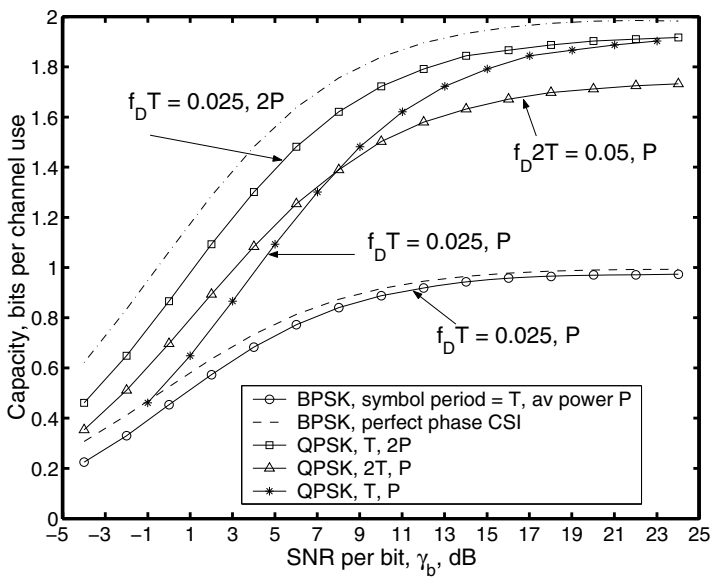

Fig. 7. Comparison of BPSK and QPSK schemes in time varying fading channels. If the average transmitted power is kept the same for both BPSK and QPSK, it is better to keep the QPSK energy per bit as high as possible in low SNR conditions. In high SNR conditions, the emphasis should be on minimizing QPSK symbol duration for minimum fading rate. 